\title{
Efficient p-n junction-based thermoelectric generator that can operate at extreme temperature conditions
}

Chavez, Ruben ; Angst, Sebastian ; Hall, Joseph ; Maculewicz, Franziska ; Stoetzel, Julie; Wiggers, Hartmut ; Le, Thanh Hung; Van Nong, Ngo; Pryds, Nini; Span, Gerjard

Total number of authors:

13

Published in:

Journal of Physics D: Applied Physics

Link to article, DOI:

$10.1088 / 1361-6463 / a a 9 b 6 a$

Publication date:

2017

Document Version

Peer reviewed version

Link back to DTU Orbit

Citation (APA):

Chavez, R., Angst, S., Hall, J., Maculewicz, F., Stoetzel, J., Wiggers, H., Le, T. H., Van Nong, N., Pryds, N., Span, G., Wolf, D. E., Schmechel, R., \& Schierning, G. (2017). Efficient p-n junction-based thermoelectric generator that can operate at extreme temperature conditions. Journal of Physics D: Applied Physics, 51(1), [014005]. https://doi.org/10.1088/1361-6463/aa9b6a

\section{General rights}

Copyright and moral rights for the publications made accessible in the public portal are retained by the authors and/or other copyright owners and it is a condition of accessing publications that users recognise and abide by the legal requirements associated with these rights.

- Users may download and print one copy of any publication from the public portal for the purpose of private study or research.

- You may not further distribute the material or use it for any profit-making activity or commercial gain

- You may freely distribute the URL identifying the publication in the public portal 
ACCEPTED MANUSCRIPT

\section{Efficient pn-junction-based thermoelectric generator that can operate at extreme temperature conditions}

To cite this article before publication: Ruben Chavez et al 2017 J. Phys. D: Appl. Phys. in press https://doi.org/10.1088/1361-6463/aa9b6a

\section{Manuscript version: Accepted Manuscript}

Accepted Manuscript is "the version of the article accepted for publication including all changes made as a result of the peer review process, and which may also include the addition to the article by IOP Publishing of a header, an article ID, a cover sheet and/or an 'Accepted

Manuscript' watermark, but excluding any other editing, typesetting or other changes made by IOP Publishing and/or its licensors"

This Accepted Manuscript is @ 2017 IOP Publishing Ltd.

During the embargo period (the 12 month period from the publication of the Version of Record of this article), the Accepted Manuscript is fully protected by copyright and cannot be reused or reposted elsewhere.

As the Version of Record of this article is going to be / has been published on a subscription basis, this Accepted Manuscript is available for reuse under a CC BY-NC-ND 3.0 licence after the 12 month embargo period.

After the embargo period, everyone is permitted to use copy and redistribute this article for non-commercial purposes only, provided that they adhere to all the terms of the licence https://creativecommons.org/licences/by-nc-nd/3.0

Although reasonable endeavours have been taken to obtain all necessary permissions from third parties to include their copyrighted content within this article, their full citation and copyright line may not be present in this Accepted Manuscript version. Before using any content from this article, please refer to the Version of Record on IOPscience once published for full citation and copyright details, as permissions will likely be required. All third party content is fully copyright protected, unless specifically stated otherwise in the figure caption in the Version of Record.

View the article online for updates and enhancements. 


\section{Efficient pn-junction-based thermoelectric generator that can operate at extreme temperature conditions}

Ruben Chavez ${ }^{1,2,}{ }^{*}$, Sebastian Angst ${ }^{1}$, Joseph Hall ${ }^{1,4}$, Franziska Maculewicz ${ }^{1}$, Julia Stoetzel ${ }^{1}$, Hartmut Wiggers ${ }^{1}$, Le Thanh Hung ${ }^{3}$, Ngo Van Nong ${ }^{3}$, Nini Pryds ${ }^{3}$, Gerhard Span ${ }^{5}$, Dietrich E. Wolf $^{1}$, Roland Schmechel ${ }^{1}$, Gabi Schierning ${ }^{1,6}$

${ }^{1}$ Center for Nanolntegration Duisburg-Essen (CENIDE), University of Duisburg Essen

${ }^{2}$ Department of Applied Physics, University of Technology Eindhoven

${ }^{3}$ Department of Energy Conversion and Storage, Technical University of Denmark

${ }^{4}$ University of lowa

${ }^{5}$ O-Flexx Technologies GmbH, Auf der Hoehe 49, D-47059 Duisburg

${ }^{6}$ Institute for Metallic Materials, IFW Dresden, Helmholtzstr. 20, D-01069 Dresden, Germany

*r.chavez@tue.nl

In many industrial processes a large proportion of energy is lost in the form of heat. Thermoelectric generators can convert this waste heat into electricity by means of the Seebeck effect. However, the use of thermoelectric generators in practical applications on an industrial scale is limited in part because electrical, thermal, and mechanical bonding contacts between the semiconductor materials and the metal electrodes in current designs are not capable of withstanding thermal-mechanical stress and alloying of the metal-semiconductor interface when exposed to the high temperatures occurring in many real-world applications. Here we demonstrate a concept for thermoelectric generators that can address this issue by replacing the metallization and electrode bonding on the hot side of the device by a p-n junction between the two semiconductor materials, making the device robust against temperature induced failure. In our proof-of-principle demonstration a p-n junction device made from nanocrystalline silicon is at least comparable in its efficiency and power output to conventional devices of the same material and fabrication process, but with the advantage of sustaining high hot side temperatures and oxidative atmosphere. 


\section{Introduction}

Thermoelectric devices can be used for solid state cooling using the Peltier effect or to produce electrical energy from heat using the Seebeck effect. The latter could be very useful for harvesting waste heat from the combustion of fossil fuels [1], the production of steel [2] or as renewable energy sources using sun light $[3,4]$. However, thermoelectric generators (TEGs) are often not able to withstand the extreme temperature conditions occurring in many of such applications. For example, although solar concentrators can reach temperatures above several thousand Kelvin, application scenarios are limited to a few hundred Kelvin in order to prevent damage to the TEGs [3], and a similar scenario is present in the steel industry where waste heat temperatures can reach more than $1500 \mathrm{~K}[2]$.

TEGs are usually made from a series of intercalated $p$ - and $n$ - type semiconductors to which ohmic contacts are realized by deposition of a metal on the semiconductor to bond it to the substrate. A TEG therefore suffers from instability due to solid state reactions at the metal-semiconductor interface at high temperatures, temperature losses at the substrate or package carrier [5], thermal stress due to the different expansion coefficients of the metal and semiconductor at the interface and thermal stress due to the temperature gradient in the semiconductor material [6]. In addition to these issues an oxidative or corrosive atmosphere leads to further problems that block the way towards practical applications of thermoelectric devices as heat engines.

A device architecture that alleviates the issues just mentioned is based on a large area p-n junction TEG (PN-TEG) in which the semiconductor materials are directly bonded together at the hot side and the electrical contacts are made exclusively on the cold side of the device (Fig. 1). Although not really common in the thermoelectric community, this device architecture was demonstrated occasionally as alternative to the standard TEG. Zakhidov et al. [7] demonstrated a thermopower enhancement in an array of pn junctions. A metal-semiconductor transition in iron disilicide was shown to effectively replace the hot side metallization in devices operated at high enough hot side temperatures, also in oxidizing atmosphere $[8,9]$. These devices were optimized in terms of geometry and performed best using U-shaped PN legs with a solid bridge between $\mathrm{p}$-type and $\mathrm{n}$-type thermoelectric materials that was pushed into a metallic state by the applied temperature. A similar device concept was realized for space missions, where Si-Ge radioisotope TEGs where equipped with a so-called hot shoe that connected the n-type and p-type material directly, only separated by a thin isolating glas layer [10]. Span et al. $[11,12]$ proposed this concept for thin-film PN-TEGs. In a study based on simulation they suggested that a PN-TEG architecture does not only help to solve technological challenges but can also be used to improve the heat to electricity conversion efficiency by the thermal generation of 
electron-hole pairs within a depletion zone $[13,14]$. Following these propositions, the role of nonequilibrium charge carriers for thermoelectricity was discussed in Ref. [15].

The use of a PN-TEG mitigates the problem of degradation or break-down of the hot side metallization upon long term high temperature operation and during cycling [16]. The mechanisms of this break down depend on the combination of materials but is usually connected to a mismatch of the coefficients of thermal expansion between the materials in contact, mechanically induced failure, chemical instability and diffusion of atoms at the interfaces.

Within this article, we provide an experimental demonstration and characterization of a PN-TEG device based on nanocrystalline silicon, which operates at conditions where conventional TEGs made by same materials and processing break down, and has at least comparable conversion efficiency as conventional TEGs. We demonstrate the functionality of the device under extreme conditions such as open flames and high temperatures reaching $1043 \mathrm{~K}$. The findings may pave the way for industrialscale applications where thermoelectric conversion under extreme conditions is needed and provide a stepping stone to investigate out of equilibrium phonons and charge carriers within a $p$-n junction.

\section{Methods}

\section{1 p-n junction thermoelectric generator (PN-TEG) based on nanocrystalline silicon} For the proof-of-principle device demonstration in this study we used nanocrystalline silicon as a thermoelectric material but in principle other materials can also be used. The main advantage of silicon as active thermoelectric converter material compared with other thermoelectric materials is that it forms a stable native oxide when exposed to high temperatures and oxidative environment. The resulting high temperature stability makes it attractive and naturally suitable for device applications under extreme environmental conditions.

The drawback of monocrystalline bulk silicon is that its thermoelectric figure of merit, $z T=\alpha^{2} \sigma T / \kappa$ where $\alpha, \sigma, \kappa, T$ are the Seebeck coefficient, electrical conductivity, thermal conductivity and temperature, is small compared to conventional thermoelectric materials because of its high thermal conductivity. This drawback can partly be tackled by the transition from micrcrystalline to a nanocrystalline bulk, first shown by the ball-milling and hot pressing approach using Bi-Te-Se ingots [17]. In silicon, this transition from microcrystalline to nanocrystalline bulk has been shown to reduce the lattice thermal conductivity up to $90 \%$ with respect to a single crystal by increased phonon scattering at grain boundaries [18]. This reduction in thermal conductivity resulted in an improvement of $z T$ from approximately 0.2 to approx 0.7 at $1250 \mathrm{~K}$ [18] for n-type nanocrystalline silicon and, in our slightly different process (see Methods: Nanoparticle production) to $z T \sim 0.6$ for $n$ type at $1250 \mathrm{~K}$ and $z T^{\sim} 0.3$ for $p$-type at $1000 \mathrm{~K}$ nanocrystalline silicon $[19,20]$. 


\subsection{Nanoparticles Production}

The nanostructured PN-TEG is prepared in a bottom-up approach. The process begins with the synthesis of highly doped silicon nanoparticles. The $n$-type nanoparticles are produced in a microwave plasma reactor using a mixture of silane $\left(\mathrm{SiH}_{4}\right)$ and phosphine $\left(\mathrm{PH}_{3}\right)$ gases. The doping concentration is tuned by adjusting the ratio of phosphine to silane. A nominal doping concentration of $1.5 \%$ results in a donor concentration of $\mathrm{N}_{A}=7.510^{20} \mathrm{~cm}^{-3}$. In a similar fashion the p-type nanoparticles are produced in a hot wall reactor that is fed with a mixture of silane and diborane $\left(\mathrm{B}_{2} \mathrm{H} 6\right)$ gases. The nominal doping concentration of the p-type nanoparticles is $2 \%$ which yields $N_{D}=1010^{20} \mathrm{~cm}^{-3}$. The produced nanoparticles have diameters that range from 10-100 nm. For more specific details on the production and properties of the $n$-type and $p$-type nanoparticles see references $[19,21]$.

\subsection{Device Assembly}

In order to produce a bulk, large area $p-n$ junction a current assisted sintering method (also known as spark plasma sintering) is used by sintering a layer of $p$-type nanoparticles on top of a layer of $n$-type nanoparticles. A constant uniaxial pressure of $35 \mathrm{MPa}$ is applied during the sintering process. A temperature of $1200^{\circ} \mathrm{C}$ is reached with a heating rate of $30 \mathrm{~K} \mathrm{~min}^{-1}$ and then held for $5 \mathrm{~min}$. For more details about the preparation of the nanostructured pn junctions see references $[22,23]$. The densified $\mathrm{p}-\mathrm{n}$ junction is cut to a geometry of $4 \mathrm{~mm} \times 3 \mathrm{~mm} \times 11 \mathrm{~mm}$. The metallization on the cold side follows a method developed by Kessler et al. [24], and consists of a nickel silicide layer, followed a nickel layer, and a capping layer of silver. The workflow of this bottom-up approach is shown in Fig. 2. More than 50 PN-TEGs were fabricated this way. The statistical deviation of the internal resistance, $R_{\text {int }}$, as an indication for reproducibility is shown in the supplemental online information, S4.

\subsection{Power generation characteristics}

A single pair PN-TEG is soldered on a carrier substrate which is mounted on the measurement setup. As a heat source a silicon carbide Ohmic heater is used. The temperatures on the PN-TEG are measured with thermocouples and the heat sink consists of a water cooled steel base where the thermocouple interconnects take place and acts as a reference temperature. Measurements are performed in vacuum conditions of $0.1 \mathrm{mBar}$. A schematic and a photograph of the set-up are shown in Fig. 3.

The power generation characteristics of the PN-TEG were performed in vacuum using an in-house test system. The I-V curves at different temperature gradients were automatically measured by sweeping the load resistance using an electronic load system. The designed measurement system is allowed to set up the measuring current range from 0 to $15 \mathrm{~A}$ with the minimum $1 \mathrm{~mA}$ step size. The controlled software system was designed based on the Apache web server software (Open Source 
Software, OSS) which is able to have maximum flexible setting up of required measurements such as gas control, cycling, and long-term stability tests etc. To define the accurate temperature gradient, a thermocouple with wire diameters of $0.5 \mathrm{~mm}$ was placed on the $\mathrm{Ag}$ electrode at the hot side of the PN-TEG. The thermocouple on the hot side is also used as the process variable for the control parameters of the heater controller. The efficiency of the device was determined by the measured out power and calculated the heat flux at the cold side. The detail of the experimental setup could be seen elsewhere [25].

\section{Results}

\subsection{Optimizing geometry to achieve maximum power output}

To optimize device performance we first studied the device power output as a function of the ratio of the cutting height $h$ to the leg height $L_{Y}$ (see Fig. 1b), aiming to achieve the highest power output [8]. This is done experimentally and by a simulation based on the Onsager-de Groot-Callen theory (see supplemental S.1). The maximum output power $P_{\max }$ can be written in the linear limit as

$$
P_{\max }=\frac{V_{O C}^{2}}{4 R_{\mathrm{int}}}
$$

where $V_{O C}$ is the device open circuit voltage, and $R_{\text {int }}$ the internal resistance. To investigate the effects of the cutting length $\$ h \$$ on the output power the thermoelectric material with $p$-n junction is removed from the substrate, a cut is made along the $p-n$ junction and then the thermoelectric material with $\mathrm{p}-\mathrm{n}$ junction is re-soldered the to the metallized substrate again. In the simulation this is done by disconnecting the bonds at the $p$ - $n$ interface (see supplemental information S.1). A simulation shows that internal current loops form for a device with $h=0$ (see supplemental information S.1). These current loops lead to undesired electrical losses. Cutting along the $p-n$ junction reduces these losses at the cost of higher internal resistance. Hence, a peak is observed in both, experiments and simulations in Fig. 4 where the maximum output power at a given cut length $P_{\max }(h)$ is normalized by the power of an uncut device $P_{\max }(h=0)$. The peak in maximum power output is not sensitive to the temperature difference in the simulation. However, the experimental data shows that both the magnitude and the location of the peak vary with applied temperature difference with no apparent trend except that the experimental data for $\Delta T=400 \mathrm{~K}$ fits the model best. The reason for this is that in the model the connections between nodes are ohmic whereas in the real device electric transport takes place across a non-ideal diode. As the temperature of the experimental device rises, the non-ideal diode interface begins to see a flatband condition and responds as an ohmic linear junction, thus the experimental data for $\Delta T=400 \mathrm{~K}$ fits the model best. 
The fact that the experimental data for $\Delta T=200 \mathrm{~K}$ and $\Delta T=300 \mathrm{~K}$ does not follow a clear trend can be understood from the inhomogeneous nature of the junction of the $p$ and n-type materials which can be seen from the SEM image in Fig. 5a) and Fig. 5b) and optically induced voltage measurements in Fig. 5c). In optically induced voltage measurements the sample is scanned with a laser source of $532 \mathrm{~nm}$, and FWHM of $20 \mu \mathrm{m}$ and the output voltage measured at a given set of $x, y$ coordinates. Fig. 5c) shows that in the area of the p-n junction a large and inhomogeneous voltage signal appears, which relates to the localized optical generation and separation of charge carriers within a depletion zone. The inhomogeneous nature of the junction arises from the mixing of the nanoparticle powders prior and during the densification step.

\subsection{Increased temperature leads to improved performance}

Despite the fact that in a PN-TEG the semiconductor materials are connected by a p-n junction which in principle is electrically isolating, the PN-TEG exhibits a very low internal resistance when the temperature is increased, thermal carrier generation lowers the potential barrier at the junction. The measured internal resistance $R_{\text {int }}$ shown in Fig. 6 has the following form:

$$
R_{\mathrm{int}}(T) \propto \exp \left(\frac{-E_{\text {activation }}}{k_{B} T}\right)
$$

where $k_{B}$ is the Boltzmann constant and $E_{\text {activation }}$ is the average activation energy of the device. In an ideal diode, the $E_{\text {activation }}$ would be $E_{g} / 2 \leq E_{\text {activation }} \leq E_{g}$, (where $E_{g}$ is the bandgap) depending on whether transport is dominated by recombination within the space charge region or diffusion of minority charge carriers. The activation energy of the PN-TEG is $\sim 230 \mathrm{meV}$, much lower than the bandgap of silicon which is $1.12 \mathrm{eV}$. The low activation energy arises from a high density of intraband states, which in turn are created during the mixing of the nanoparticle powders prior and during the sintering process. The low activation energy is beneficial for the operation of the PN-TEG because a quasi-ohmic contact is created at the $p$-n junction when the temperature on the hot side is increased. Unlike conventional TEGs where $R_{\text {int }}$ increases linearly with temperature [26], in a PN-TEG $R_{\text {int }}$ decreases exponentially with temperature until the equivalent of a flat band condition is reached. After $T_{h}$, the hot side temperature reaches $686 \mathrm{~K}$ saturation is observed in $R_{\text {int }}$ in Fig. 6 indicating that the equivalent of a flat band condition has been reached (see insets in Fig. 6) and hence the top part of the device behaves as close as possible to an ohmic contact.

\subsection{Performance comparison between PN-TEGs and conventional TEGs}

A side by side comparison between a PN-TEG and a conventional TEG built in the same lab by the same source materials and processing shows that higher temperature differences can be applied to the PN-TEG and that the output power and efficiency of a PN-TEG can be equal or superior to a conventional TEG. The output power of a PN-TEG is compared to a conventional TEG simulated in 
ideal conditions (no electrical or thermal contact resistances), and also compared to an experimental conventional TEG that was fabricated from the same source materials and methods [24]. In Fig. 7, we do this comparison by means of a normalized output power $P_{0}$ which is defined as

$$
P_{0}=\frac{P_{\max } L}{n A}
$$

where $n$ is the number of thermocouples, $A$ the cross-sectional area of one thermocouple and $L$ the length of the semiconductor legs. In Fig. 7, $P_{0}$ of a nanocrystalline silicon PN-TEG with the optimal cutting ratio $h / L_{\gamma}=0.73$ is shown. The simulation of the conventional TEG is fed with measured temperature dependent values of $\alpha, \sigma$, and $\kappa$ from the same materials used to make the PN and conventional TEGs $[19,20]$ and included in Fig. 7.

The offset between the TEG simulation data and TEG experimental data in Fig. 7 arises from the fact that the simulation contains no parasitic electrical and thermal contact resistances, whereas the measured device clearly included these losses decreasing the power output by about one order of magnitude. Since the conventional TEG has a limited range in operational temperature, a fit proportional to $\Delta T^{2}$ is shown as a dashed line.

The PN-TEG shows a poor performance in the lower temperature regime in Fig. 7, orders of magnitude lower than the conventional TEG. This is because the diode limits the current flow. As the temperature difference is increased, the diode approaches the flat band mode and the PN-TEG outperforms the experimental conventional TEG and approaches the simulation of an ideal TEG with no parasitic losses. This shows that a PN-TEG offers clear advantage not only in higher temperature stability but also in better performance compared to a conventional TEG.

We next performed efficiency measurements by applying a controlled temperature difference to the PN-TEG and measured the heat flow and output power [25]. Fig. 8a) shows sample current-voltage measurements for the PN-TEG under different temperature differences where the linear response of the PN-TEG can be observed and with cold side temperature fixed at approximately $300 \mathrm{~K}$. Fig.8b) compares the PN-TEG with an ideal (no electrical and thermal contact resistances) conventional TEG using measured $\alpha(T), \sigma(T), N(T)$ which is the maximum possible efficiency for the given materials and it is shown that the PN-TEG reaches about $60 \%$ of the maximum possible efficiency. The maximum efficiency of a PN-TEG reaches $0.57 \%$ at $T_{h}=823 \mathrm{~K}$, compared to the efficiency of a previously reported conventional TEG built by the same source materials and processing which had a maximum efficiency of $0.25 \%$ at $T_{h}=823 \mathrm{~K}$ [24]. In both cases the cold side was coupled to cooling water at approximately $300 \mathrm{~K}$. The higher efficiency of the PN-TEG at even lower hot side temperature than that of the conventional TEG clearly demonstrates the advantage of the design of the PN- TEG, which minimizes the thermal and electrical losses on the hot side. 
Since the PN-TEG does not require a hot side substrate and metallization it could hereby be characterized up to $1043 \mathrm{~K}$ on the hot side temperature (see supplemental information S.3) which is considerably higher than the maximum hot side temperature of $873 \mathrm{~K}$ reported on the conventional TEG [24] showing how a thermoelectric module can benefit from the PN-TEG architecture.

Moreover we show that a PN-TEG can be operated in harsh environment like open flames. We have exposed a series connection of four PN-TEGs to a Bunsen burner as heat source while the cold side was water cooled. A sketch is shown in Fig.9, and a video showing the experiment can be found in the supplemental information. While conventional TEGs fail this experiment, a PN-TEG based on silicon suffers no damage. Even though oxidation occurs at the hot side of the PN-TEG, this does not create a device failure since no metal-semiconductor interface is affected. In fact, exposing the silicon PN-TEG at the hot side to high-temperatures and corrosive operation conditions, just produces a capping layer of silicon oxide which makes the device even more robust.

\section{Conclusion}

We have shown that a thermoelectric generator made with a $p$ - $n$ junction on the hot side can reach higher efficiency than a counterpart made with traditional ohmic contacts on the hot side. The $p-n$ junction on the hot side makes the PN-TEG more robust against temperature induced failure such as alloying or diffusion at the metal-semiconductor interface. Hence, higher temperatures and harsh conditions such as open flames can be applied on the hot side of a PN-TEG showing how the findings here reported can have practical applications. The concept of a PN-TEG is demonstrated with nanocrystalline silicon, but other materials, especially those prepared by spark plasma sintering can benefit from this approach. We believe that the concept of a PN-TEG can be further improved by tunning the activation energy, for example by having higher doping concentration on the hot side of the thermoelectric leg. This work open up a new and unexplored way of designing and using TEG.

\section{Acknowledgments}

Financial support in the frame of a young investigator Grant by the Ministry for innovation, science and research of the State North Rhine Westphalia in Germany is gratefully acknowledged. Further, financial support by the German Research Foundation within the priority program SPP 1386 on nanostructured thermoelectrics is gratefully acknowledged. 


\section{References}

[1] Bell, L.E., Cooling, Heating, Generating Power, and Recovering Waste Heat with Thermoelectric Systems. Science, 2008. 321(5895): p. 1457-1461.

[2] Kuroki, T., et al., Thermoelectric generation using waste heat in steel works.J. Electr. Mater., 2014. 43(6): p. 2405-2410.

[3] Kraemer, D., et al., High-performance flat-panel solar thermoelectric generators with high thermal concentration. Nat. Mater., 2011. 10(7): p. 532-538.

[4] Olsen, M.L., et al., A High-temperature, High-efficiency Solar Thermoelectric Generator Prototype. Energy Proc., 2014. 49: p. 1460-1469.

[5] Apertet, Y., et al., Optimal working conditions for thermoelectric generators with realistic thermal coupling. Europhys. Lett., 2012. 97(2): p. 28001.

[6] Ziabari, A., E. Suhir, and A. Shakouri, Minimizing thermally induced interfacial shearing stress in a thermoelectric module with low fractional area coverage. Microelectronics Journal, 2014. 45(5): p. 547-553.

[7] Zakhidov, A.A., Y.I. Ravich, and D.A. Pchenoy-Severin. Thermopower enhancement and optimal ZT in $p$-n junction arrays. in Thermoelectrics, 1999. Eighteenth International Conference on. 1999.

[8] Volklein, F., W. Wittmer, and U. Birkholz, Analysis of the optimum leg length of a thermoelectric generator with a directly sintered hot junction. Energy Convers. Management, 1993. 34(8): p. 687-690.

[9] Stohrer, U., et al., Sintered FeSi2 for thermoelectric power generation. Energy Convers. Management, 1990. 30(2): p. 143-147.

[10] Hartman, R.F. and C.E. Kelly, MOD-RTG multicouple test results and mission readiness. AIP Conf. Proc., 1993. 271(1): p. 301-306.

[11] Wagner, M., et al., Thermoelectric power generation using large-area Si/SiGe pn-junctions with varying Ge content. Semicond. Sci. Techn., 2007. 22(1): p. S173.

[12] Span, G., et al. Thermoelectric Power Conversion using Generation of Electron-Hole Pairs in Large Area p-n Junctions. in 25th International Conference on Thermoelectrics, ICT '06. 2006.

[13] Melnick, C. and M. Kaviany, Phonovoltaic. I. Harvesting hot optical phonons in a nanoscale $\$ p \mid$ text \{lensuremath\{-\}\}n $\$$ junction. Phys. Rev. B, 2016. 93(9): p. 094302.

[14] Span, G., et al., Miniaturized TEG with thermal generation of free carriers. Phys. Status Solidi (RRL), 2007. 1(6): p. 241-243.

[15] Gurevich, Y.G. and J.E. Velázquez-Pérez, The role of non-equilibrium charge carriers in thermoelectric cooling. J. Appl. Phys., 2013. 114(3): p. 033704.

[16] Ebling, D., et al., Module Geometry and Contact Resistance of Thermoelectric Generators Analyzed by Multiphysics Simulation. J. Electr. Mater., 2010. 39(9): p. 1376-1380.

[17] Poudel, B., et al., High-Thermoelectric Performance of Nanostructured Bismuth Antimony Telluride Bulk Alloys. Science, 2008. 320(5876): p. 634-638.

[18] Bux, S.K., et al., Nanostructured Bulk Silicon as an Effective Thermoelectric Material. Adv. Mat., 2009. 19(15): p. 2445-2452.

[19] Kessler, $V_{\text {., }}$ et al., Thermoelectric properties of nanocrystalline silicon from a scaled-up synthesis plant. Adv. Eng. Mat., 2013. 15(5): p. 379-385.

[20] Schierning, G., et al., Role of oxygen on microstructure and thermoelectric properties of silicon nanocomposites. J. Appl. Phys., 2011. 110(11): p. 113515-9.

[21] Petermann, N., et al., Plasma synthesis of nanostructures for improved thermoelectric properties. J. Phys. D, 2011. 44(17): p. 174034.

[22] Meseth, M., et al., The realization of a pn-diode using only silicon nanoparticles. Script. Mat., 2012. 67(3): p. 265-268. 
[23] Becker, A., et al., A sintered nanoparticle p-n junction observed by a Seebeck microscan. J. Appl. Phys., 2012. 111(5): p. 054320-7.

[24] Kessler, V., et al., Fabrication of high-temperature-stable thermoelectric generator modules based on nanocrystalline silicon. J. Electr. Mat., 2014. 43(5): p. 1389-1396.

[25] Hung, L.T., et al., Segmented Thermoelectric Oxide-Based Module for High-Temperature Waste Heat Harvesting. Energy Technology, 2015. 3(11): p. 1143-1151.

[26] Niu, X., J. Yu, and S. Wang, Experimental study on low-temperature waste heat thermoelectric generator. Journal of Power Sources, 2009. 188(2): p. 621-626.

[27] Chavez, R., et al., High temperature thermoelectric device concept using large area pn junctions. J. Electr. Mat., 2014. 43(6): p. 2376-2383. 


\section{Figures and Figure Captions}

\section{Figure 1}

a) Conventional TEG

b) PN -TEG

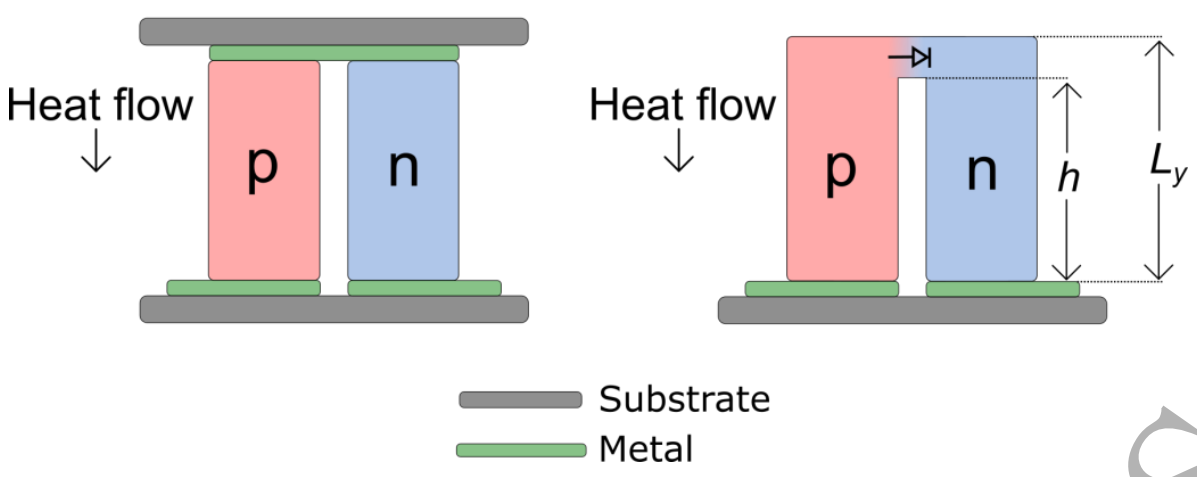

Figure 1) Schematics of the device setup for conventional and PN-TEG architectures. a) In a conventional TEG the p-type and n-type semiconductor materials which comprise the active device are connected electrically in series using a metal interconnect. The active device is then mounted on a substrate which is often made of ceramic. b) In a PN-TEG the metal contact and substrate on the hot side are dismissed, and the electrical connection made by a $p-n$ junction of the semiconductor materials. The dismissal of the metal contacts and substrate on the hot side in a PN-TEG proves to be a formidable technological advantage. 


\section{Figure 2}

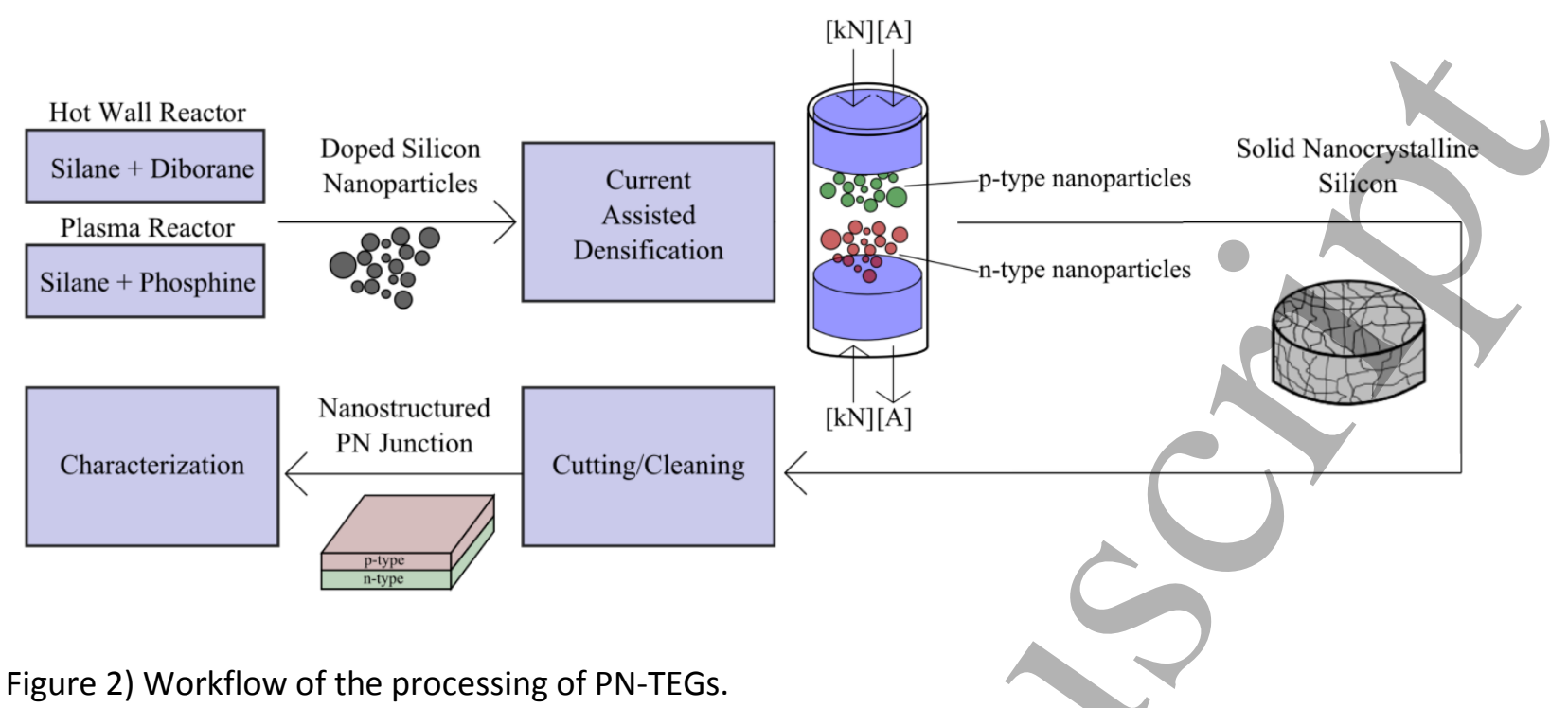

Figure 2) Workflow of the processing of PN-TEGs. 


\section{Figure 3}
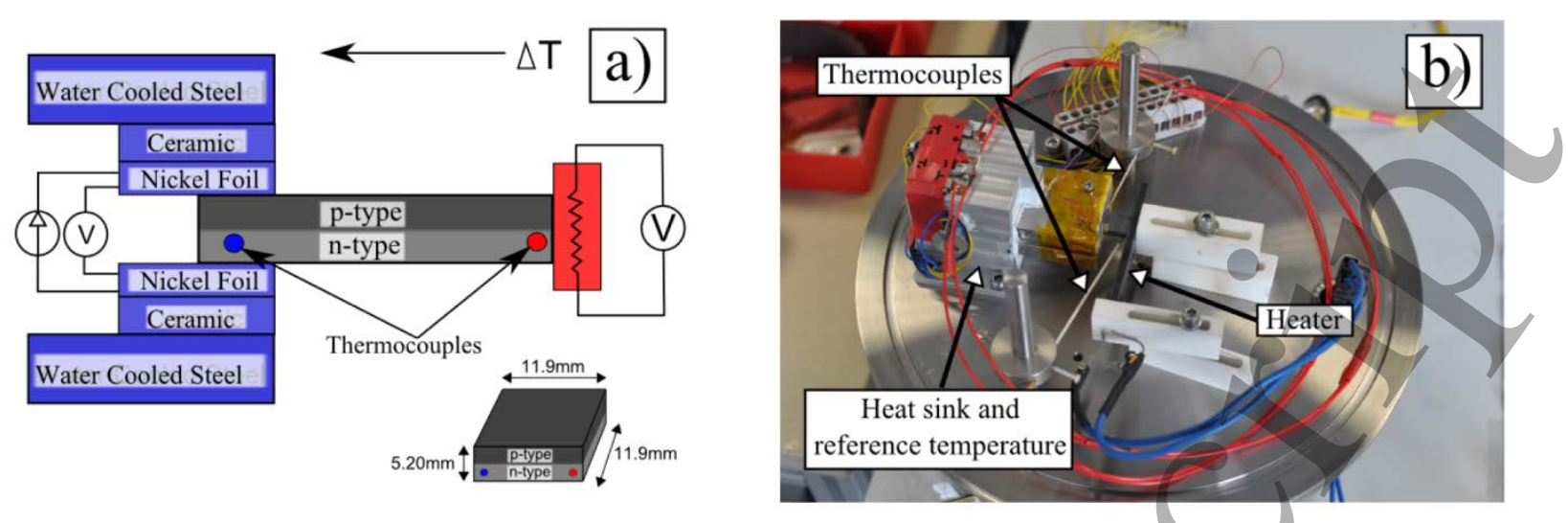

Figure 3a) Schematic of the set-up for the characterization electrical output data of PN-TEGs with varying hot side temperature. Reproduced with permission, Ref.[27], Copyright 2014, Springer. b) Photograph of the actual setup. 


\section{Figure 4}

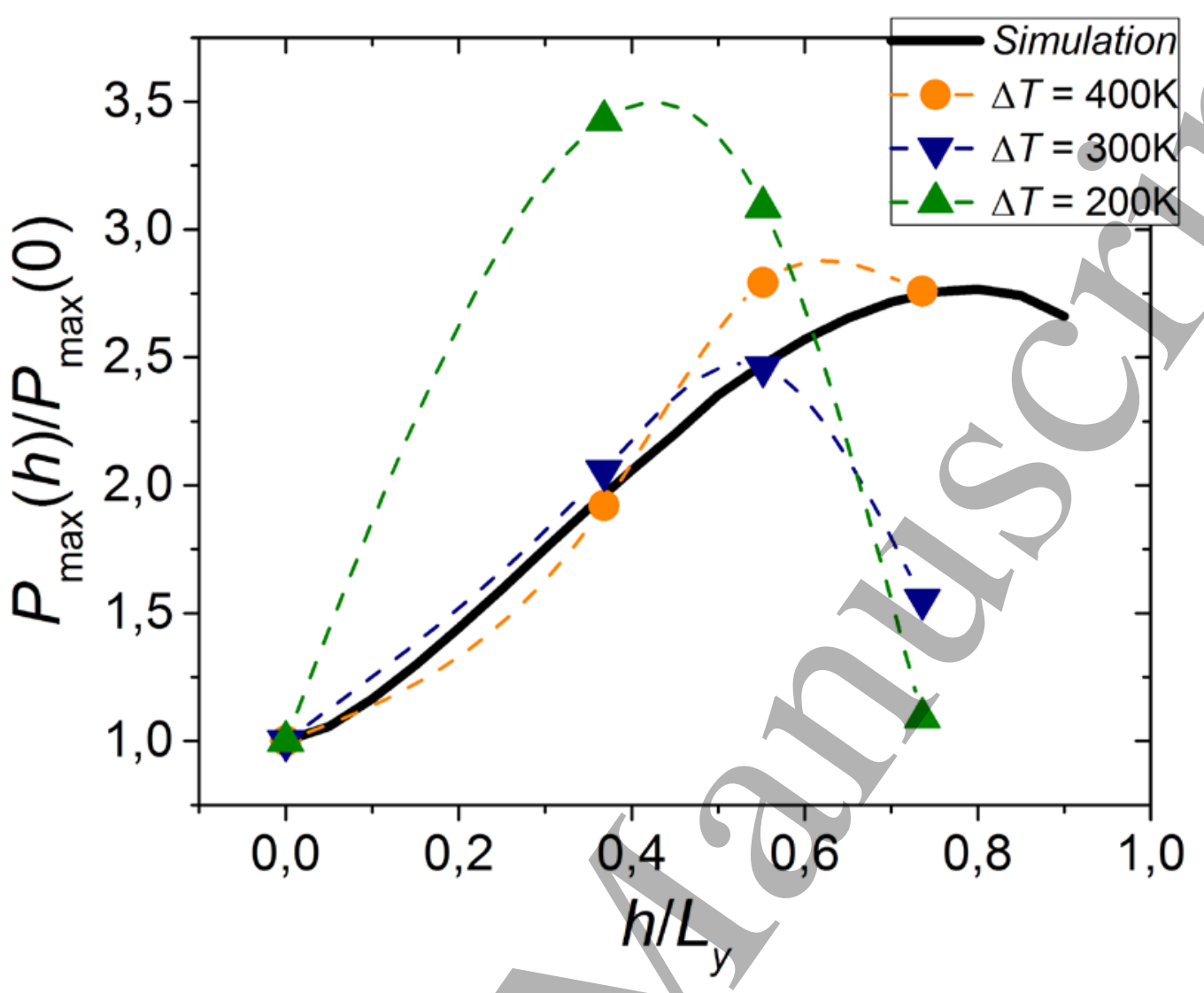

Figure 4) Output power gain factor in dependency of the ratio of the cutting height and the length of the device $h / L_{\gamma}$. A maximum gain in output power is predicted by the simulation and confirmed by the experiments. 


\section{Figure 5}

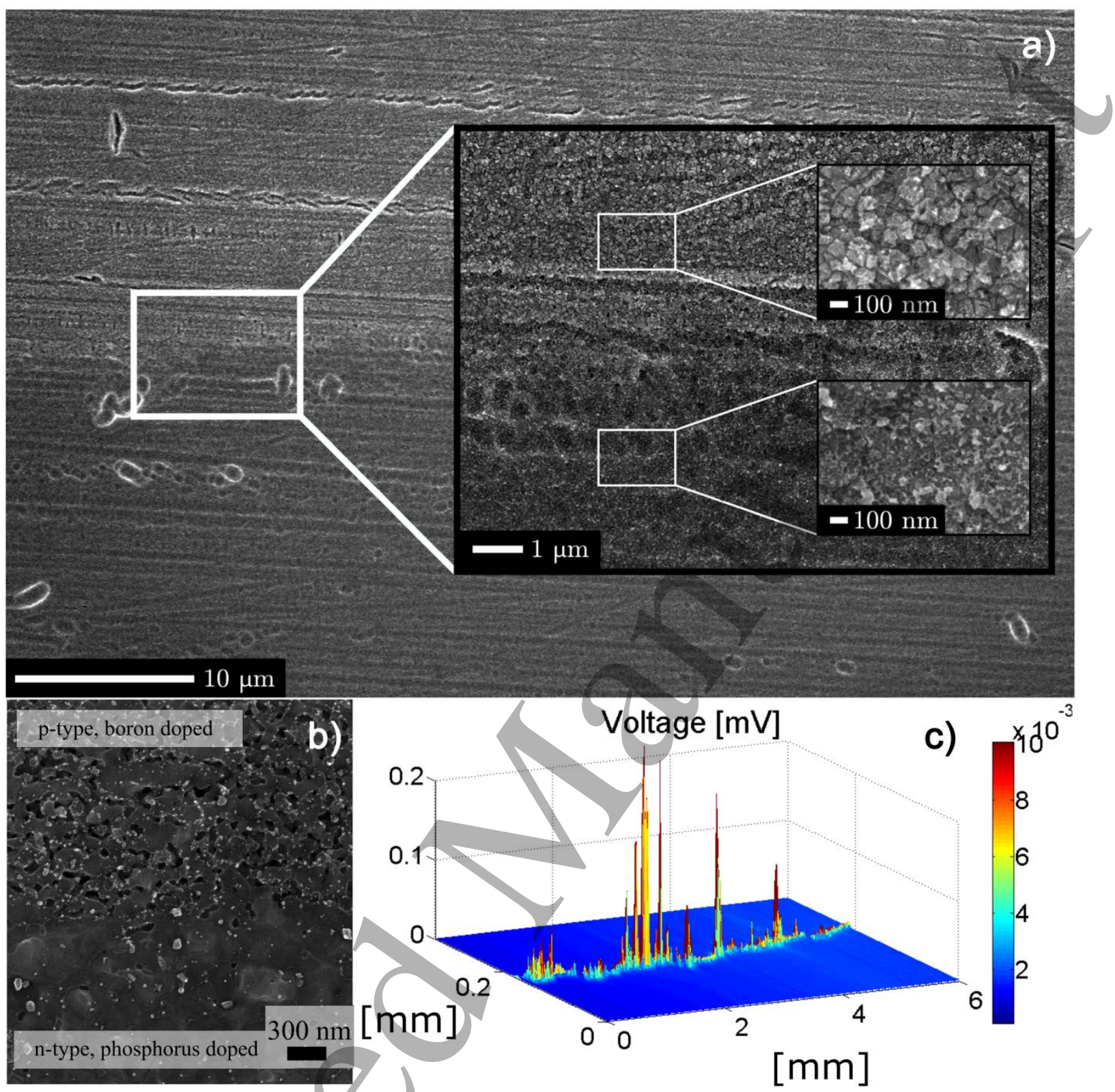

Figure 5a) SEM image of the p-n junction. The sample was dipped in hydrofluoric acid prior to imaging; boron doped nanocrystalline silicon develops more agglomeration of $\mathrm{SiO}_{\mathrm{x}}$ and thus appears more porous. b) Zoom-in of $\mathrm{p}$-n junction region of the sample. c) Optically induced voltage measurements which can be correlated to domains where charge generation and separation is successful, show the inhomogeneous nature of the $p$-n junction. (c) reproduced with permission from Ref. [27], Copyright 2014, Springer. 
Figure 6

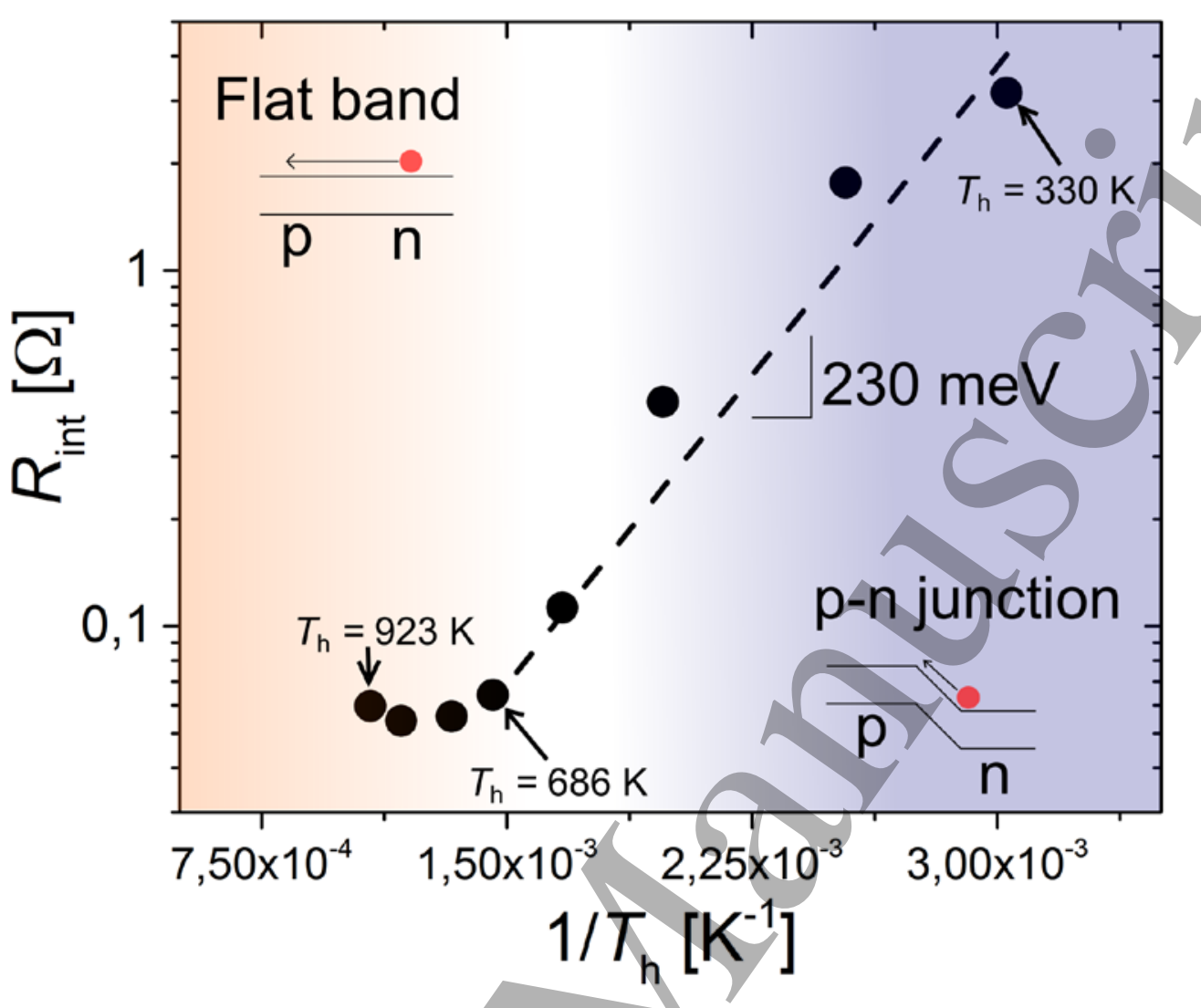

Figure 6) Temperature dependency of $R_{\text {int }}$ with temperature shows that a flat band condition is reached for temperatures higher than $686 \mathrm{~K}$. 


\section{Figure 7}

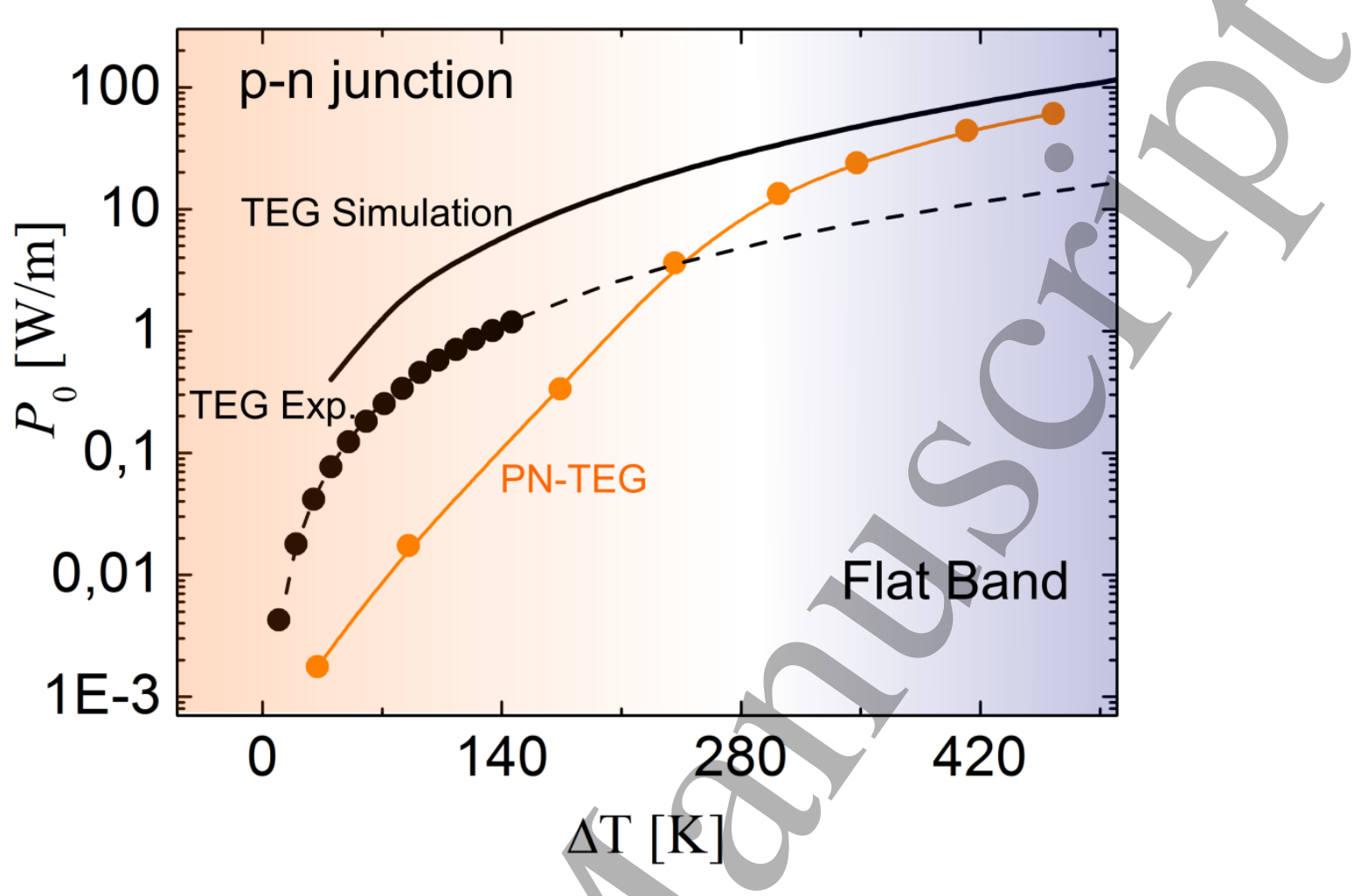

Figure 7) Normalized output power of a PN-TEG and a conventional TEG made from the same materials and process, as well a simulation of an ideal conventional TEG shows that PN-TEG performs best at high temperature differences. 


\section{Figure 8}
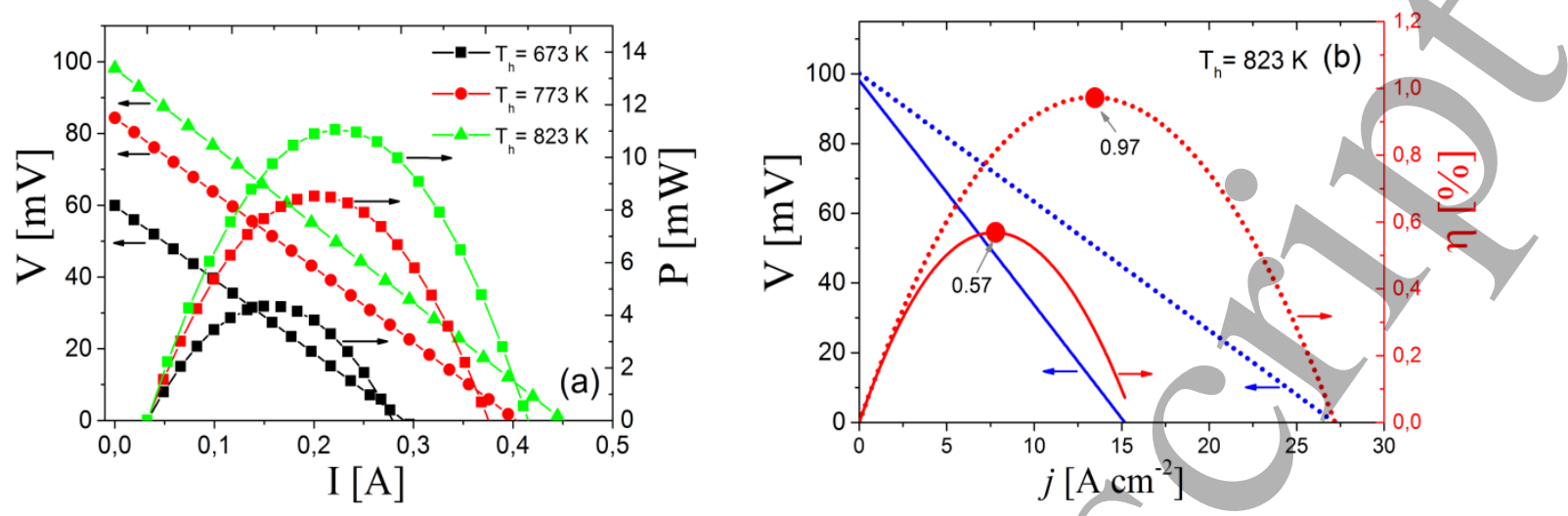

Figure 8(a) Output voltage and output power for the PN-TEG as a function of current under different hot side temperatures show the linear response of the PN-TEG. (b) Experimental $V(j)$ and efficiency of the PN-TEG (solid lines) compared with the calculation of a conventional TEG under ideal conditions (dotted lines). The maximum efficiency of a conventional TEG made of the same materials and processing reaches $0.25 \%$ at $T_{h}=873 \mathrm{~K}$ [24]. The cold side in all cases was coupled to cooling water at approximately $300 \mathrm{~K}$. 


\section{Figure 9}

a)

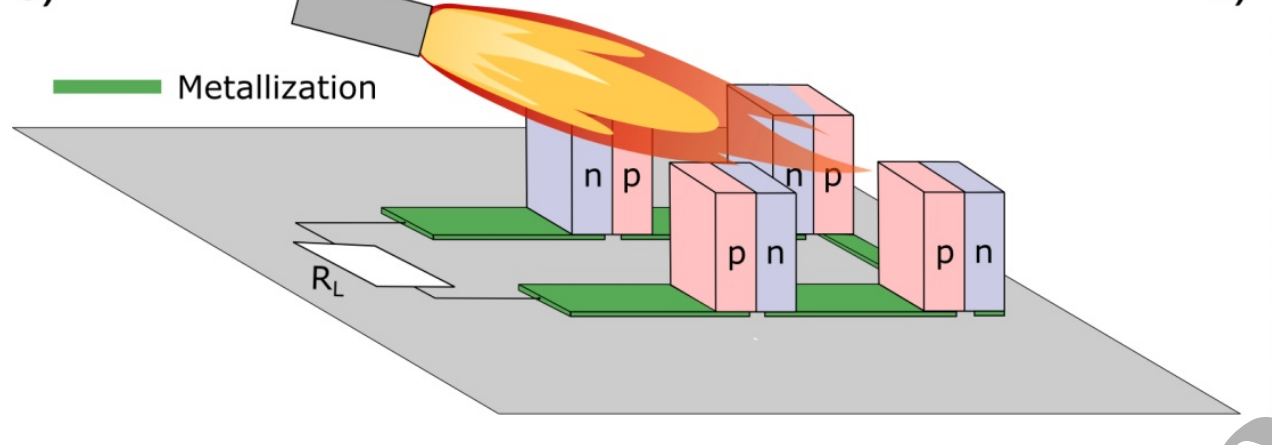

b)

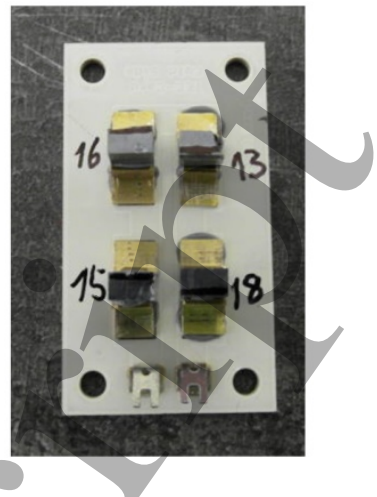

Figure 9) Open flame operation of a series connection of 4 PN-TEGs made of nanocrystalline silicon. The cold side is water cooled. a) Sketch of the experiment. b) Photograph of the experimental realization (see supplemental information for video). 